\title{
TDMA based HR-WPAN Application for a Ship Area Network with Link Transmission Parameters Selection Algorithm
}

\author{
Dong-Keun Jeon ${ }^{1}$ and Yeonwoo Lee ${ }^{2 \dagger}$ \\ ${ }^{1}$ Department of Mechatronics Engineering, School of Mechanical Engineering, \\ Incheon National University, Incheon, Korea \\ E-mail:dkjeon@incheon.ac.kr \\ ${ }^{2}$ Department of Information and Communications Engineering, \\ College of Engineering, Mokpo National University, Chonnam, Korea \\ E-mail:ylee@mokpo.ac.kr
}

\begin{abstract}
For a high-speed data transmission with guaranteed QoS and energy efficiency in a ship area network (SAN), we consider IEEE 802.15.3 based TDMA high-rate wireless personal area network (HR-WPAN) application. It is shown that the proposed SAN architecture with TDMA based HR-WPAN can provide a high quality wireless connectivity to the conventional dedicated wired devices and shipboard control network devices as a wireless gateway, by adaptively selecting transmission parameters such as transmission power and physical data rate for a fixed fragment size. Moreover, a realistic effect of ship building material such as steel (large ship) and FRP (medium size ship) is considered as a path loss parameter for performance evaluation. We demonstrated that the proposed TDMA based HR-WPAN system with the link transmission parameters selection algorithm could provide a good wireless network model applicable to the SAN architecture.
\end{abstract}

Keywords: Ship Area Network, Energy Efficiency, HR-WPAN, Shipboard Control Network, QoS

\section{Introduction}

The typical ship area network (SAN) architecture of the international standard is mainly based on wired networks such as dedicated connections, instrument networks and shipboard control networks (Ethernet based connection). This network delivers main operations such as sensing and control shipboard systems and management of crucial information for safety and navigation. These operations are performed in many parts of the vessel from the engine room, to the bridge, to the administrative personnel, and even off of the ship to the owner's office. The general SAN architecture is generally organized as a single network with a backbone network comprised of three levels, i.e., instrument level, process level and system level. The lowest level is an instrument level in which instruments, devices and sensors are connected. A process level deals with a number of processes that interconnect devices and computers. The top layer in the trusted part of the system is the Integrated Ship Control (ISC) layer. This is used to interconnect different process segments as required and also to bridge over to administrative functions where this is needed. As the ISC layer potentially connects to all process segments, one single error on the ISC layer may propagate down into several systems if no safeguard is put in place. Thus, one will need some form of gateway (GW) or Firewall (FW)

${ }^{\dagger}$ Corresponding author 
between the ISC layer and each of the process segments [1]. This hierarchical structure with three levels is standardized by the international maritime organization (IMO) or the international electro-technical commission (IEC) [2].

However, the maximum throughput of the navigational instrument's bus supported by the current controller area network (CAN) connection is limited to $125 \mathrm{kbps}$. With this, a typical CAN cannot satisfy the increasing need for large amount of data transmission on board between a bunch of instruments and an integrated gateway. Besides control and navigational information between instruments devices, the need for various data services within a vessel is essential for a high-value added vessel. For this, a wireless transmission between devices (sensors) and a gateway is a reasonable option with respect to energy efficiency, system deployment cost, and recovery and management convenience. As one of such research works, a wireless gateway applying WiMedia distributed MAC protocol and transmission diversity was proposed and its performance evaluation was presented in [3]. This work had been based on relay operation in WiMedia distributed MAC systems proposed in [4] as well as energy efficient transmission scheme proposed in [5].

On the other hand, for a higher cost benefit vessel, i.e. digital ship, many researchers recently have been focusing on developing an integrated SAN applying state of the art IEEE 802.15.x based wireless communication technologies such as wireless sensor network (WSN), wireless personal network (WPAN) as well as WiMedia based SAN network as in [3]. As one of recent research works, the SAN architecture with TDMA based WPAN had been presented in [6], which dealt with a conceptual machine-tomachine (M2M) network model. However, this work could not suggest a detailed operation and performance evaluation results of TDMA based HR-WPAN system.

Thus, this paper presents a TDMA based HR-WPAN application model to satisfy the need for various data services with energy efficiency for a ship area network. As a wireless transmission of a wireless gateway, HR-WPAN piconet coordinator (PNC) is chosen because it can guarantee energy efficient operations with high date rate transmission in a wireless network environment. If the HR-WPAN is operated in conjunction with a TDMA based MAC protocol, the system parameters in each allocated time slot such as transmission power and physical layer (PHY) data rate can be adjusted to minimize energy consumption and satisfy the QoS requirements of each device. Section 2 in this paper describes the proposed SAN architecture with TDMA based HRWPAN PNC and its traffic model. In Section 3, the TDMA based HR-WPAN system model and its link parameter selection algorithm are presented. Section 4 shows the simulation results and discussion of the proposed system model, followed by the conclusion.

\section{Ship Area Network Architecture with TDMA based HR-WPAN}

\subsection{Conventional Ship Area Network Architecture}

An integrated navigation system on board the ship using National Marine Electronics Association (NMEA) 2000 standard is primarily designed to provide two-way communication between the ship's navigational equipment such as radar, GPS receiver, automatic identification system (AIS), Gyro compass etc. NMEA 2000 is based on CAN, which is standardized by ISO. NMEA 2000 standard also became the international standard under IEC. ISO 11898 specifies physical and data-link layer of serial communication technology called CAN that supports distributed real-time control and multiplexing. The protocol is used to create a network of electronic devices, mainly marine instruments, on a vessel. Various instruments with the NMEA standard are connected to one central wired cable; i.e., a backbone. The backbone powers each instrument and relays data among all of the instruments on the network [7].

Navigational instruments are normally interconnected with the well-established IEC 61162-1 or IEC 61162-2 standards. These are based on standard asynchronous serial lines 
with a text message protocol. Communication speeds vary from $4.8 \mathrm{kbps}$ (IEC 61162-1) up to $38.4 \mathrm{kbps}$ (IEC 61162-2). The network uses a single talker and a number of listeners in a star-shaped configuration. The standard is also referred to as NMEA 0183 that is the basis for the IEC-produced specification. However, for Safety of Life At Sea (SOLAS) certified ships, the correct reference to use is IEC 61162 [2]. NMEA 2000 is IECaccepted as a standard IEC 61162-3 on IEC technical committee 80, working group 6 (digital interfaces), and successfully develops another LAN (Local Area Network) standard for ships where LAN requires high capacity and also high security (IEC 611624) [7]. This system will allow up to about 50 nodes to share a common bus at $250 \mathrm{kbps}$. Messages vary from 8 bytes up to about 1,000, but at an overhead of $50 \%$. Thus, effective maximum throughput on the bus is about $125 \mathrm{kbps}$. Note that these navigational instruments need to be interconnected both on the integrated bridge system that is for navigational system integration based on Ethernet protocol.

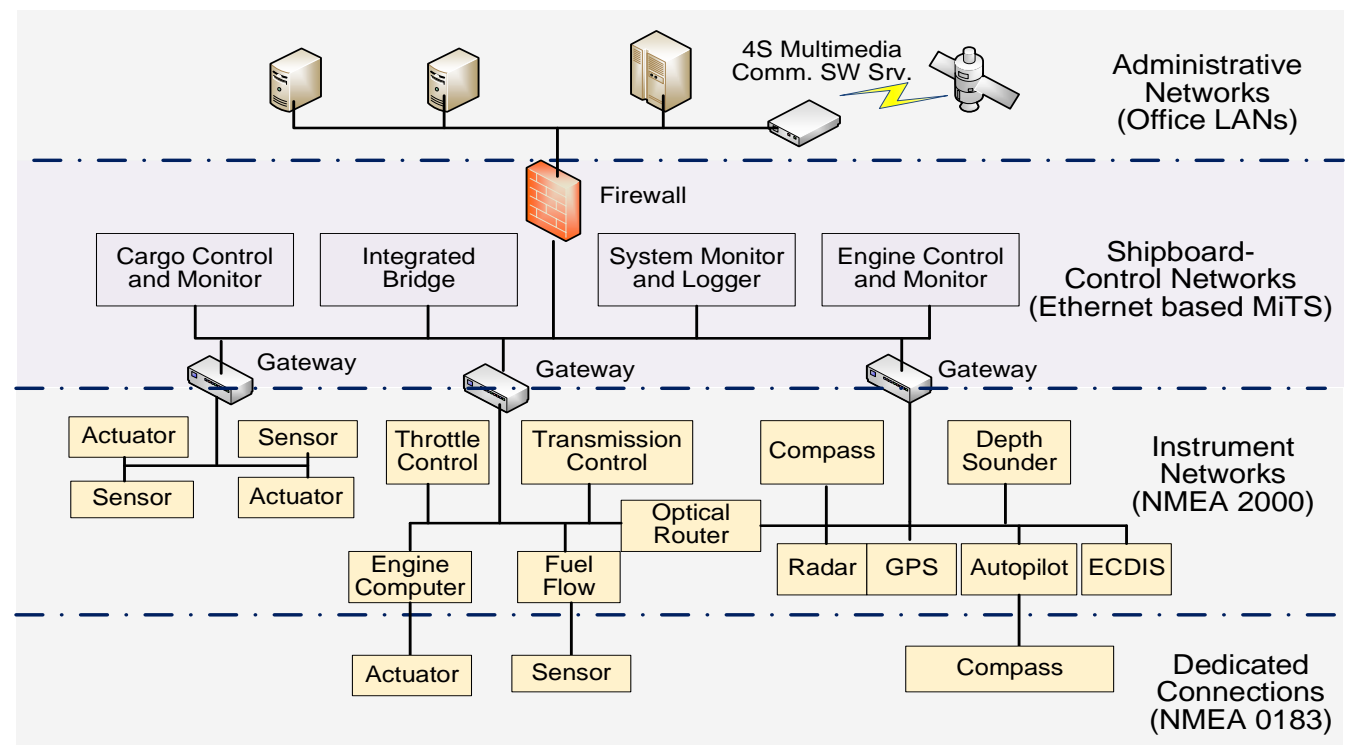

\section{Figure 1. Conventional SAN Architecture based on International Standards [7]}

The first version of shipboard data architecture developed by the IEC standard is the Maritime Information technology Standard (MiTS) project [1, 7]. This uses four layers: instrument, process, system, and administrative. The MiTS was developed as an integrated ship control (ISC) protocol, which could integrate an NMEA network on the bridge with industrial data network in the automation system. The conventional ship area network architecture is shown in Figure 1.

\subsection{SAN Architecture with TDMA based HR-WPAN Piconet System}

As mentioned before, data throughput of the conventional SAN is not sufficient to satisfy the increasing amount of data caused by a bunch of various machines, devices, and sensors. Therefore, such a high data rate supported system is necessary for providing a degree of freedom in deploying infra-nodes and reliable networking with many instruments and devices at the lowest level. Thus, this paper proposes TDMA based HRWPAN with WPAN piconet network coordinator (PNC) and devices as shown in Figure 2, wherein a HR-WPAN PNC acts as a wireless gateway between a machine (instrument, device, or sensor) and a ship board control network (i.e., wired Ethernet network) of the SAN. In the proposed architecture, each WPAN device (DEV) is a wireless transceiver connected to each navigational instrument or sensors, as shown in Figure 2. 


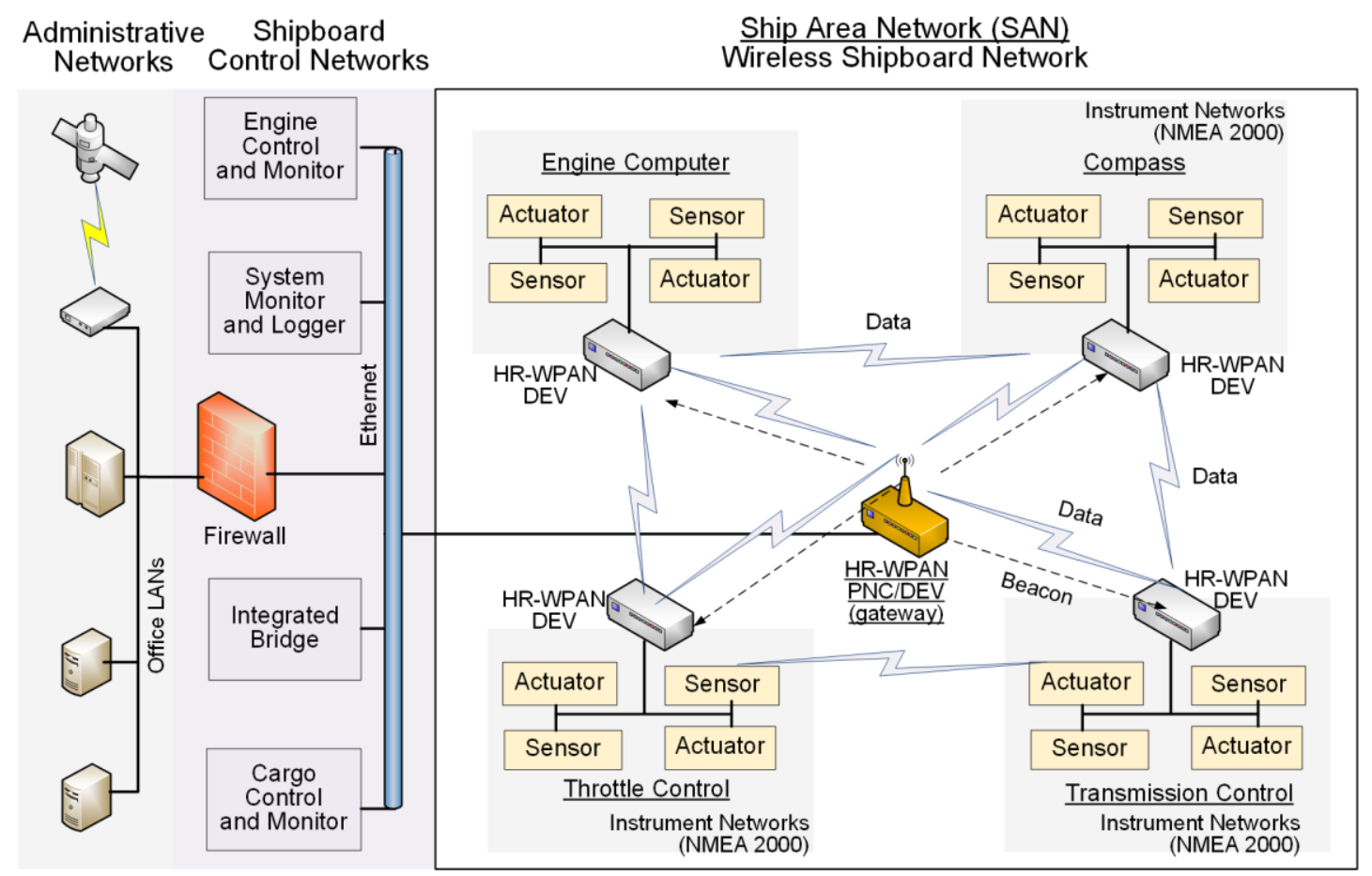

Figure 2. The Proposed SAN Architecture of TDMA based HR-WPAN System

For this, it is noted that the WPAN piconet should be capable of supporting high-speed data transmission with high quality and energy efficiency with devices. Thus, in this paper, TDMA based MAC protocol is applied to the HR-WPAN system model of the proposed SAN architecture. As shown in Figure 2, a HR-WPAN PNC/DEV is capable of providing the timing beacon signal and managing the QoS for the HR-WPAN piconet of the SAN architecture. Each HR-WPAN DEV is connected to NMEA 2000 instruments with functionality of transmission control, throttle control, engine computer and compass. For high throughput support with guaranteed energy efficiency and QoS, this HR-WPAN piconet should adaptively select the link transmission parameters such as transmission power and PHY data rate in each allocated time slot. Thus, the proposed HR-WPAN piconet can minimize energy consumption and satisfy the QoS requirements of each device. Consequently, the proposed SAN architecture with TDMA-based HR-WPAN successfully transmits, controls, and/or manages information from a central shipboard control network station to/from devices or instruments.

\subsection{Traffic Model of the SAN Architecture}

The number of devices in the SAN of a general container ship is almost 460 and these devices are connected by an isolated network [8]. These devices are connected to the SAN and transmit traffics through the network as shown in Figure 1 and Figure 2. Most data traffic within a vessel carry control and status information and thus, traffic must be lossless with low latency allowance. The traffic model of the SAN architecture considered in this paper is as shown in Table 1, wherein the packet size, transmission frequency and data amount of the traffic are not ignorable because these traffic are represented in GPS, integrated navigation system (INS), voyage data recorder (VDR), AIS, gauge NN1 (the device for measuring balance of the ship), and Web server PC. It is noted that in this paper the traffic model is mainly based on Table 1. Furthermore, the traffic generated from instrument sources such as throttle control, transmission control, engine computer and compass is also considered in this paper, along with this traffic model. 
Table 1. Traffic Model of the SAN Architecture [8]

\begin{tabular}{cccc}
\hline Source & $\begin{array}{c}\text { Transmission } \\
\text { frequency (message } \\
\text { per second) }\end{array}$ & $\begin{array}{c}\text { Message size } \\
\text { (bytes) }\end{array}$ & Number of devices \\
\hline \hline GPS & 1 & $79 \sim 200$ & $2 \sim 15$ \\
INS & 50 & 79 & $2 \sim 15$ \\
VDR & 1 & 79 & $5 \sim 15$ \\
AIS & 50 & $79 \sim$ & $2 \sim 15$ \\
Gauge NN1 & 1 & 79 & $5 \sim 15$ \\
PC (Web server) & 1 & 79 & $5 \sim 15$ \\
\hline
\end{tabular}

\section{TDMA based HR-WPAN System and Link Parameter Selection}

\subsection{IEEE 802.15.3 based TDMA HR-WPAN System Model}

In this paper, IEEE 802.15.3 HR-WPAN piconet model is assumed, whereof a piconet coordinator (PNC) is supposed to provide the timing with the beacon signal and manage the QoS for the piconet. In the superframe of the HR-WPAN system standard model, the Contention Access Period (CAP) is used to communicate commands or asynchronous data and the Contention Free Period (CFP) is reserved for channel time allocations (CTAs), which are used for commands, isochronous streams and asynchronous data connections. During the CTA, a device can transmit data without any interference from other devices in the same piconet. Thus, the CTA is useful for carrying data frames with specific QoS provisions. In this paper, it is assumed that a TDMA-based HR-WPAN system supports 3 data rates, i.e., 11, 22 and 33Mbps with QPSK-TCM, DQPSK-TCM and 16QAM-TCM, respectively [9].

\subsection{Link Transmission Parameter Selection Algorithm}

The energy required to transmit a frame using fragment size $L$, data rate (i.e., modulation level) $R$ and transmission power $P_{t}$, is given by $E_{\text {frame }}\left(L, R, P_{t}\right)=T_{\text {frame }}(L, R) P_{t x}\left(P_{t}\right)$ where $T_{\text {frame }}$ is the transmission time of the frame. And $P_{t x}$ is the total power required to transmit the frame and is defined as $P_{t x}=P_{c_{-} \text {mod }}+P_{t} / \eta$ where $P_{t}$ is the output power of the transmitter in the strict sense, $\eta$ represents the efficiency of the power amplifier, and $P_{c_{-} \text {mod }}$ denotes the power consumed in the modulation circuit of the transmitter including the other baseband processors. The total power consumption of a device in receive mode, $P_{r x}=P_{c_{-} \text {demod }}$ $+P_{r}$ where $P_{r}$ is the power consumed at the receiving front end and $P_{c_{-} \text {demod }}$ denotes the power consumed in the circuit of the demodulator including the other baseband processors. The power consumed in the modulation $\left(P_{c_{-} m o d}\right)$ circuits and that in the demodulation $\left(P_{c_{-} \text {demod }}\right)$ circuits are set to the same value, $P_{c}[10]$. When considering the transmission of an entire MAC service data unit (MSDU), with a size of $L_{M S D U}$, a device must transmit $\left\lceil L_{M S D U} / L\right\rceil$ frames. Therefore, the energy required to transmit an entire MSDU without consideration on transmission errors is obtained as follows.

$$
\begin{aligned}
& E_{M S D U}\left(L, R, P_{t}\right) \\
& =\left\lfloor\frac{L_{M S D U}}{L}\right\rfloor \cdot E_{\text {frame }}\left(L, R, P_{t}\right)+E_{\text {frame }}\left(L_{\text {remain }}, R, P_{t}\right) \\
& +\left\lceil\frac{L_{M S D U}}{L}\right\rceil \cdot\left(E_{\text {SIFS }}+E_{A C K}\right)
\end{aligned}
$$


where $L_{\text {remain }}$ is the length of the remaining data after the MSDU has been fragmented by splitting it into $L$ fixed size fragments. Based on Eq. (1), the total energy consumed by a device during an assigned CTA period is given in [10]. It is assumed that a CTA is assigned to each node based on the amount of data each node needs to transmit. The total energy consumed for the transmission of $K$ MSDUs during the CTA period is given by

$$
E_{C T A}=\sum_{k=1}^{K} E_{M S D U, k}\left(L, R, P_{t}\right)
$$

where

$$
\begin{aligned}
E_{M S D U, k}\left(L, R, P_{t}\right)= & \left(\left\lfloor\frac{L_{M S D U, k}}{L}\right\rfloor \cdot E_{\text {frame }}\left(L, R, P_{t}\right)+E_{\text {frame }}\left(L_{\text {remain }}, R, P_{t}\right)\right. \\
& \left.+\left\lceil\frac{L_{M S D U, k}}{L}\right\rceil\left\{\left(E_{\text {SIFS }}+E_{A C K}\right)(1-F E R)+E_{\text {RIFS }} \cdot F E R\right\}\right) \cdot \sum_{i=0}^{q} F E R^{i}
\end{aligned}
$$

as explained in [10].

The physical parameters selection algorithm is designed to minimize energy consumption by determining the optimal combination of fragment size, data rate and transmission power, which are based on measurement information of received signal strength indicator (RSSI) and link quality indicator (LQI) [10]. The selection algorithm is to find the parameter combination of $L$ (fixed fragmented size of MAC data unit), $R$ (data rate) and $P_{t}$ (transmission power), i.e., $\left[L, R, P_{t}\right]$, which is subject to minimize the consumed energy for the duration of channel time allocation (CTA), $E_{C T A}$. If both noise power and path loss are constant for the duration of CTA period, maintaining the combination $\left[L, R, P_{t}\right]$ during CTA period will be the best strategy. However, since the noise power and path loss may not be constant during CTA duration in a wireless channel, we must update the optimal set into the new values of $\left[L, R, P_{t}\right]$ so as to minimize the total transmission energy for the remaining time duration in CTA. It is noted that in the proposed HR-WPAN piconet based SAN architecture, the path loss are very dependent on material of the ship. Thus, in our simulation, various path loss values are considered in order to take into account the effect of material of the ship.

\section{Simulation Results and Discussion}

As a simulation platform, we use Matlab for the performance evaluation of the proposed architecture of SAN with TDMA based HR-WPAN. Most parameters assumed in this simulation are based on IEEE 802.15.3 physical parameter and energy parameters [9] and we mainly simulate CFP with several CTA periods. It is assumed that a fragment size is fixed to 1024 bytes and the maximum transmission power is between 0 and $10 \mathrm{dBm}$ with step size of $2 \mathrm{~dB}$. The other parameters are given in Table 2. We assume that the size of MSDUs transmitted during CTA of 0.006 seconds is 3000 bytes, and 10 HR-WPAN devices connect shipboard instruments are randomly distributed in SAN piconet model.

Without realistic channel measurement, it is assumed that the path loss of ship environment would be higher than that of terrestrial environment and thus, in this simulation, three path loss cases such as $50 \mathrm{~dB}, 55 \mathrm{~dB}$ and $60 \mathrm{~dB}$ are considered as small ship, FRF ship, and steel ship, respectively. This is to consider the effect of material of the ship construction in our simulation. We consider three vessel conditions such as steel ship and FRF ship cases. For a steel ship, the path loss parameter is assumed as $60 \mathrm{~dB}$ and for a FRF ship, $55 \mathrm{~dB}$ is assumed. 
Table 2. IEEE 802.15.3 based HR-WPN Physical Layer Simulation Parameters [9]

\begin{tabular}{ll}
\hline \multicolumn{1}{c}{ Parameter } & \multicolumn{1}{c}{ Value } \\
\hline \hline Fragment size (bytes) & 1024 (fixed) \\
\hline$T_{A C K}$ & $22.54 \mu \mathrm{s}$ \\
\hline$P_{C}, P_{r}$ & $400 \mathrm{~mW}, 50 \mathrm{~mW}$ \\
\hline Path loss & $50 \mathrm{~dB}, 55 \mathrm{~dB}, 60 \mathrm{~dB}$ \\
\hline
\end{tabular}

The energy consumption results of the TDMA based HR-WPAN system of SAN architecture are shown in Figure 3 and Figure 4 according to variation of noise power for fixed PHY data rate and transmission power, respectively. In Figure 3, the energy consumption is very dependent on the variation of path loss value. It is noted that when path loss increases (i.e., very large amount of absorption and attenuation of the radio frequency wave by the wall within a ship; severe wireless channel condition), energy consumption abruptly increases at a certain value of noise power. For example, when noise power approaches about $-60 \mathrm{dBm}$, the consumed energy by piconet devices with fixed rate of $33 \mathrm{Mbps}$ and path loss of $60 \mathrm{~dB}$ goes to infinity.

It is noted that the energy consumption floor is very decided by choice of PHY data rate. That is, when the data rate is fixed to $11 \mathrm{Mbps}$, the minimum energy consumption of this rate is higher than that of $33 \mathrm{Mbps}$ data rate irrespective of noise power. Moreover, it can be inferred from this result that for fixed amount of data to be transmitted, the HRWPAN piconet with a higher power and data rate can save the transmission energy by virtue of link parameter selection algorithm. As such, the link parameter selection algorithm finds the near optimum parameter set in order to minimize the energy consumption and maximize the data throughput.

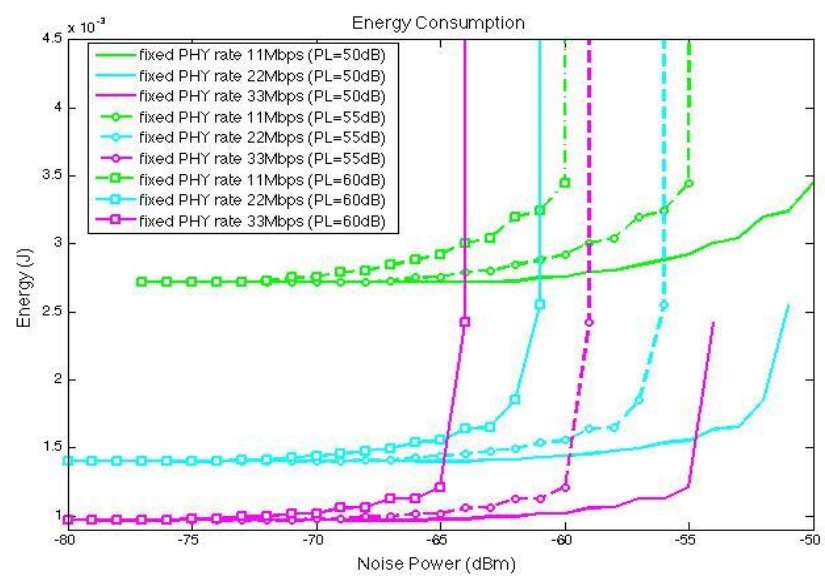

\section{Figure 3. Energy Consumption Comparison Result of TDMA based HR-WPAN SAN Architecture with Fixed PHY Data Rate Selection versus Noise Power}

As show in Figure 4, the energy consumption as function of transmission power is also subject to path loss and transmission power. For a given transmission power, we can see that a higher power and lower path loss can help to decrease energy consumption of devices. Different from the result of Figure 3, the energy consumption floor cannot be observed in Figure 4 because of step size of data rate (i.e., 11, 22 and $33 \mathrm{Mbps}$ ). If we assume that path loss of steel ship and FRF ship are $60 \mathrm{~dB}$ and $55 \mathrm{~dB}$, respectively, it is 
noted from the results of Figure 3 and Figure 4 that the overall energy consumption for steel ship is much higher than that for FRF ship.

Simulation results of both Figure 3 and Figure 4 show that the overall energy consumption for a steel ship is much higher than that for a FRF ship. However, when the noise power increases above $-65 \mathrm{dBm}$, a higher data rate transmission needs more energy for devices to communicate. In such case, the selection of a lower PHY data rate transmission is reasonable. On the other hand in Figure 4, if we fixed the PHY data rate, the higher power of devices requires the lower energy consumption as expected. This result implies that the transmission with fixed transmission power of all of devices according to the channel condition can prevent the HR-WPAN piconet from wasting the transmission energy. As shown in Figure 4, it can be seen that that if the transmission powers of all piconet devices are fixed to $8 \mathrm{dBm}$, the overall consumed energy by piconet is the lowest. This means that all devices with a fixed high transmission power can select each near-optimized data rate according to each device's channel status. Thus, while the parameter selection is very dependent on each channel status such as path loss and noise power level, the piconet with a fixed high transmission power is most reasonable for energy saving perspective.

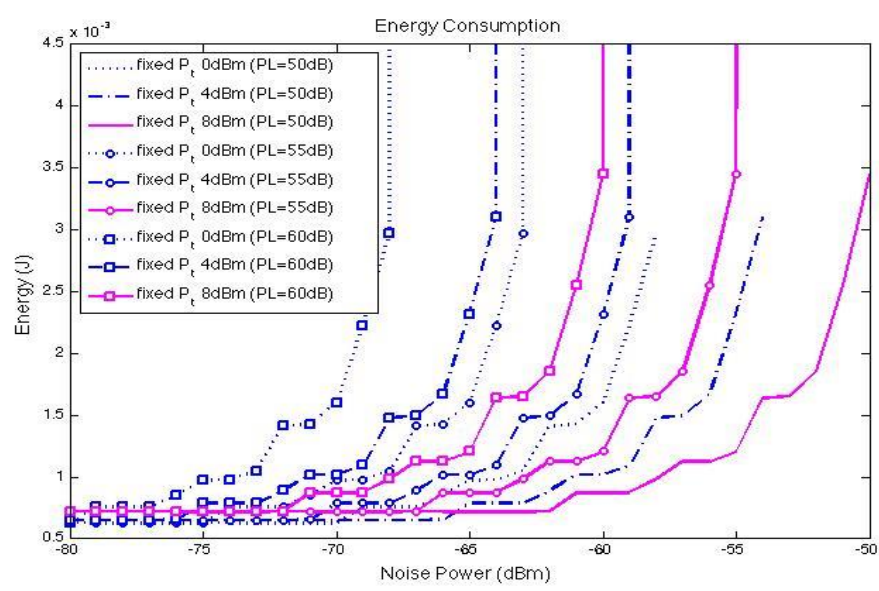

Figure 4. Energy Consumption Comparison Result of TDMA based HR-WPAN SAN Architecture with Fixed Transmission Power Selection versus Noise Power

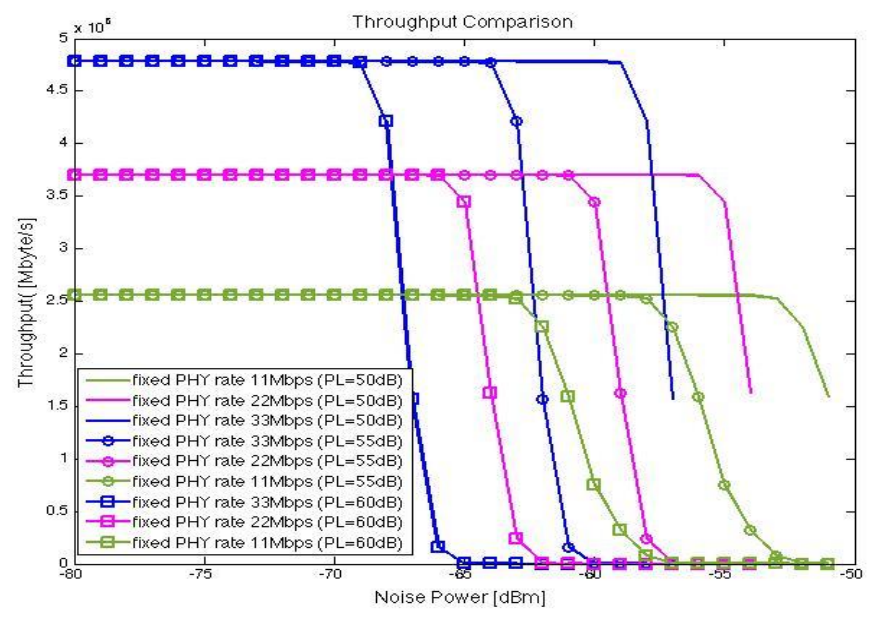

Figure 5. Throughput Comparison Result of TDMA based HR-WPAN SAN Architecture with Fixed PHY Rate Selection versus Noise Power 
Figure 5 shows the throughput comparison result as function of the noise power with PHY data rate and path loss as parameters. It can ne observed that with a higher PHY data rate or lower path loss, more data packet can be successfully delivered to the destination devices. It is also noted that when a path loss increases, throughput curve abruptly drops to floor at a certain value of noise power as similar with Figure 3. We can see that the decisive parameter of throughput performance is the PHY data rate. It means that only if channel status is favorable, the HR-WPAN piconet system wit a higher fixed PHY data rate can guarantee the maximum data throughput. Otherwise, the system cannot support any data transmission. When the data rate is fixed to 33 Mbps with path loss of $60 \mathrm{~dB}$, data cannot be delivered above the noise power level of $-65 \mathrm{dBm}$.

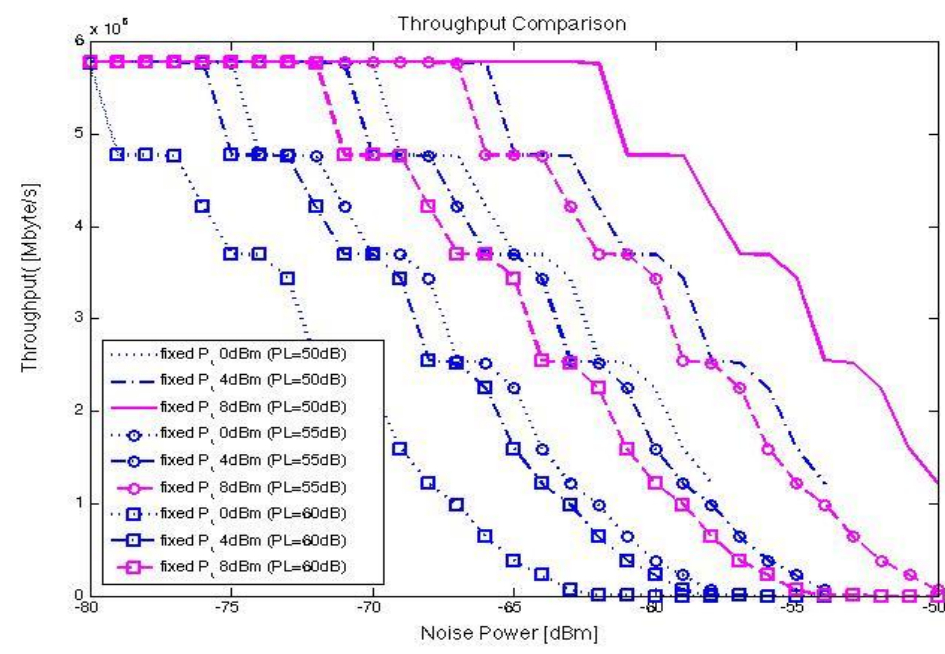

\section{Figure 6. Throughput Comparison Result of TDMA based HR-WPAN SAN Architecture with Fixed Transmission Power Selection versus Noise Power}

Figure 6 demonstrates the throughput performance comparison as function of noise power with path loss and transmission power as parameters. As similar with the previous results, it can be seen that with higher transmission powers of all devices, more data throughput can be successfully achieved by appropriate parameter selection data rate. The reason of the stepwise throughput results as shown in Figure 6 is the stepwise change of data rate (i.e., 11, 22 and $33 \mathrm{Mbps}$ ) by the link parameter selection algorithm according to channel status. It is also noted that the higher achieved data throughput can be expected when the path loss decreases. This means that the overall throughput in case of a steel ship is much lower than that in case of a FRF ship.

As similar observation with Figure 4, the HR-WPAN piconet system with a fixed high transmission power of $8 \mathrm{dBm}$ can successfully deliver a higher data throughput support to all of devices. Similar observations can be seen as that of the simulation results of Figure 4. Thus, while the parameter selection is very dependent on each channel status such as path loss and noise power level, we can see that the HR-WPAN piconet system with a sufficiently high transmission power can be most reasonable choice for guaranteeing better system performance in the context of energy consumption and data throughput from both Figure 4 and Figure 6. 


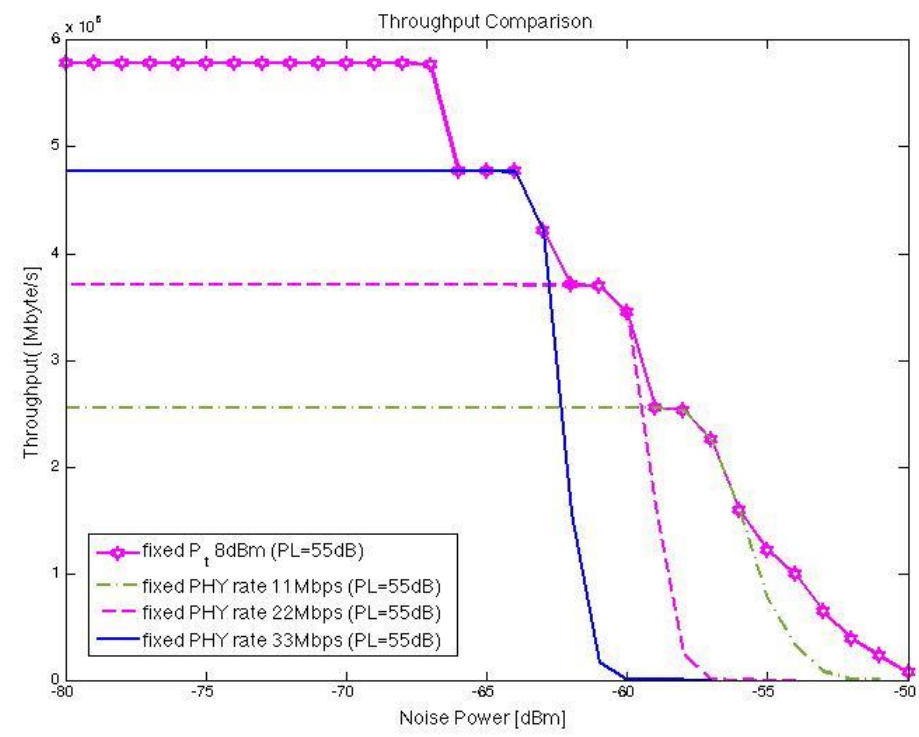

\section{Figure 7. Throughput Comparison Result of TDMA based HR-WPAN SAN Architecture with Fixed Transmission Power Selection versus Noise Power}

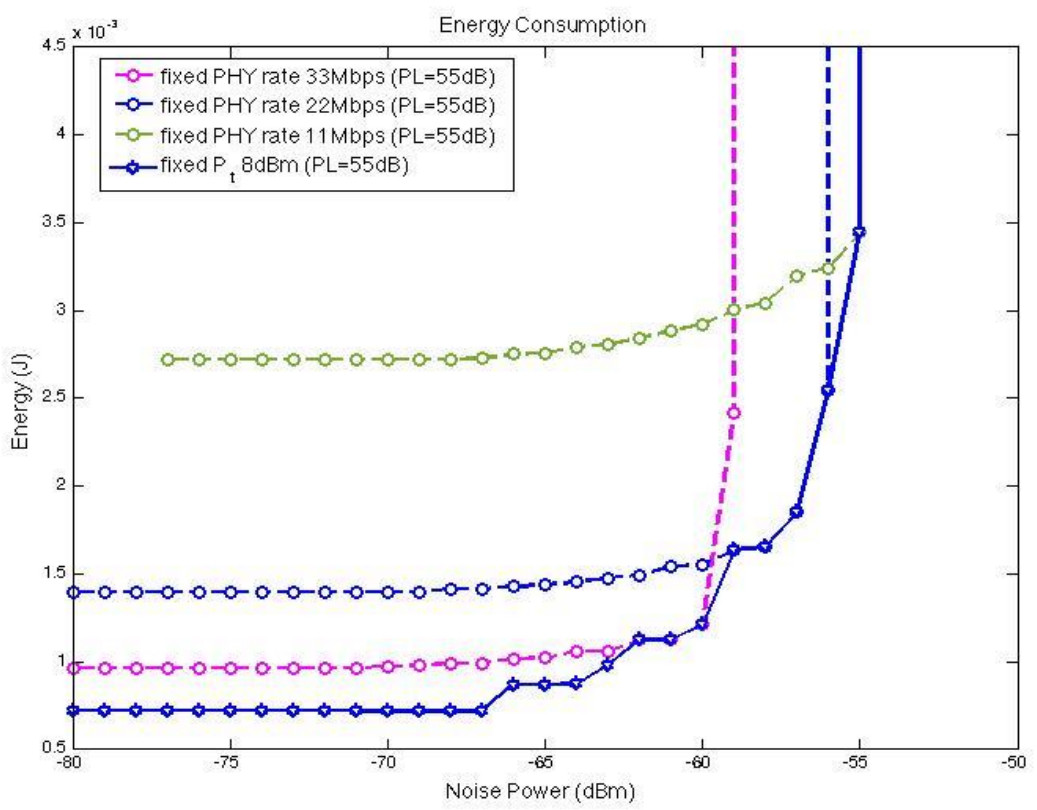

Figure 8. Energy Consumption Comparison Result of TDMA based HRWPAN SAN Architecture with Fixed Transmission Power Selection versus Noise Power

In order to compare the effect of link parameter selection irrespective of path loss, we simulated throughput performance and energy consumption performance for a given path loss value of $55 \mathrm{~dB}$ as shown in Figure 7 and Figure 8, respectively. In Figure 7, it can be observed that the throughput performance with the fixed transmission power of $8 \mathrm{dBm}$ follows the maximum throughput contour line of the simulated results with the fixed data rate. As to the energy consumption, a similar result can be observed in Figure 8. Thus, it can be concluded that the adaptively selection of data rate with sufficiently high 
transmission power can guarantee both better performance of energy consumption and data throughput. The reason of this is that for the fixed transmission power of all devices, the link parameter selection of the data rate can provide more precise performance resolution. Once the data rate is fixed, the performance resolution decided by the change of transmission power is limited because of large step size.

\section{Conclusion}

In this paper, we proposed the SAN architecture with TDMA based HR-WPAN piconet model with an adaptive link transmission parameter selection algorithm for a high-speed data transmission with guaranteed QoS and energy efficiency. It was confirmed that the proposed architecture with TDMA based HR-WPAN piconet system could guarantee the minimum energy consumption and the maximum data throughput by adaptively selecting parameter according to channel condition and path loss (i.e., ship environment). It was derived that the system with the higher fixed transmission power required the lower energy consumption and delivered the higher data throughput, although both performances were very dependent on each channel status such as path loss and noise power level. Thus, the proposed SAN with TDMA based HR-WPAN piconet system with the link parameter selection algorithm can be successfully applicable to the wireless device-to-device or instrument-to-instrument communication network deployed with ship area network environment.

\section{ACKNOWLEDGEMENTS}

This research was supported by Basic Research Program through the National Research Foundation of Korea (NRF) funded by the Ministry of Education, Science and Technology (No. 2013R1A1A2008722).

\section{References}

[1]. "Maritime Information T Standards (MiTS)", available at http://www.mits-forum.org/architecture.html.

[2]. S. Krile, D. Kezić and F. Dimc, "NMEA Communication Standard for Shipboard Data Architecture", Our Sea, International Journal of Maritime Science \& Technology, vol. 60, no. 3, (2013).

[3]. D. Jeon and Y. Lee, "Performance Evaluation of a WiMedia based Wireless Bridge using Relay Cooperative Transmission", Advanced Science and Technology Letters, (Mobile and Wireless 2014), vol. 60, (2014).

[4]. S. Lee, Y. Lee and S. Lee, "Multi-helper Relay Based WUSB/DRD/WLP Protocol in WiMedia Distributed MAC Systems", International Journal of Multimedia and Ubiquitous Engineering, vol. 9, no. 3, (2014).

[5]. S. Lee, M. Choi, Y. Lee, S. Park and J. Kim, "An Energy Efficient Multimedia Streaming Scheme in WiMedia Networks", International Journal of Multimedia and Ubiquitous Engineering, vol. 9, no. 1, (2014).

[6]. D. Jeon and Y. Lee, "Performance Evaluation of Ship Area Network with TDMA-based HR-WPAN for a M2M Application", Proceeding of The 8th International Conference on Future Generation Communication and Networking, (2014) December 20-23, Hainan, China.

[7]. Ø. J. Rødseth and E. Haaland, "MiTS-An Open Standard for Integrated Ship Control", Proceedings of ICMES, vol. 93, (1993) September, Hamburg, Germany.

[8]. S. Lee, J. Kim, K. Moon, K. Lee and J. Park, "Backbone Network Architecture Planning of Ship Area Network", Proceedings of the $7^{\text {th }}$ IEEE VTC Asia Pacific Wireless Communication Symposium (APWCS), (2010) May 20-21, Kaohsiung, Taiwan.

[9]. “IEEE 802.15.3”, Part 15.3. Wireless MAC and Physical Layer WPAN Standard, IEEE, (2003).

[10].Y. Joo and Y. Lee, "Energy-Efficient Transmission Scheme for WPANs with a TDMA-based Contention-Free Access Protocol", IEICE Transactions on Communications, vol. E91-B, no. 2, (2008). 


\section{Authors}

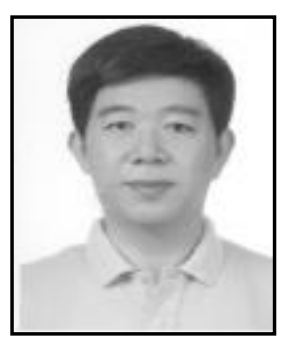

Dong-Keun JEON, he is currently a Professor with the Department of Mechatronics Engineering at the Incheon National University, Incheon, Korea. He received the B.S. degree in electronics engineering from Korea University, Seoul, Korea, in 1986 and the M.S., and Ph.D. degrees in electronics engineering from Korea University, Seoul, Korea, 1988 and 1992, respectively. His research interests include wireless communication system, mobile and satellite communications system, applications of sensor networks and mechatronics related robot system.

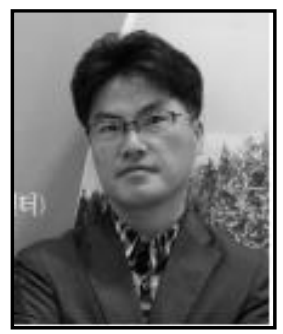

Yeonwoo LEE, he is currently a Professor with the Department of Information and Communications Engineering at the Mokpo National University, Mokpo, Korea, since September 2005. He has been a Senior Researcher with $4 \mathrm{G}$ Mobile Communication team at the Samsung Advanced Institute of Technologies (SAIT), Kiheung, from January 2004 to August 2005. From October 2000 to December 2003, he has been a Research Fellow with the School of Electronics and Engineering at the University of Edinburgh, UK. From October 2000 to December 2002, he joined core 2 work of Mobile VCE program in UK. He received a MS and Ph.D. in Department of Electronics Engineering from Korea University, Seoul Korea, in 1994 and 2000, respectively. He is currently IEEE senior member, member of IEICE and lifelong member of KICS. His research interests are wireless mobile telecommunication systems, radio resource management, (ad-hoc) multihop relay system, sensor network and cognitive radio systems. 